\title{
Las lenguas minoritarias en la Sociedad de la Información. Cartografía, revitalización y aprendizaje del aragonés en entornos virtuales
}

\section{Minority languages in the Information Society. Cartography, revitalization and learning of Aragonese on virtual environments}

\section{Dr. David García-Marín}

Universidad Rey Juan Carlos | Calle Camino del Molino, 5, 28943 Fuenlabrada | España |

https://orcid.org/0000-0002-4575-1911 | david.garciam@urjc.es

\author{
Alejandro Ibáñez-Alcázar \\ Universidad Nacional de Educación a Distancia (UNED) | Calle Juan del Rosal, 14, 28040 Madrid \\ | España | https://orcid.org/0000-0002-0947-1218 | aibanez280@alumno.uned.es
}

Fechas | Recepción: 04/07/2021 | Aceptación: 30/11/2021

Investigación financiada por el Proyecto Internética. Proyecto I+D+i subvencionado por el MCINN (PID 2019-104689RB-100). Fecha de inicio: 1 de junio 2020. Fecha de fin: 1 de junio 2023.

\section{Resumen}

El presente trabajo analiza la situación actual de la lengua aragonesa en la Red; una lengua minoritaria y minorizada que, según la UNESCO, se encuentra en peligro de extinción. Sin embargo, gracias a Internet y a la rápida expansión de la Web 2.0, las lenguas minoritarias como el aragonés cuentan con nuevas oportunidades para su revitalización. El objetivo es comprender cómo las nuevas tecnologías y el conjunto de posibilidades que ofrece la Web influyen positivamente en su visibilidad y contribuyen a evitar su desaparición. Para ello, esta investigación -de tipo eminentemente cualitativo- se desarrolla desde dos enfoques: en primer lugar, desde una perspectiva comunicativa, para hacer una aproximación a la presencia, uso y difusión del aragonés en plataformas virtuales; $y$, en segundo lugar, desde una mirada educativa, para analizar las posibilidades de aprendizaje de esta lengua en

\begin{abstract}
This paper analyzes the current situation of the Aragonese language on the Web; a minority language that, according to UNESCO, is threatened with extinction. However, thanks to the Internet and the development of Web 2.0, minority languages such as Aragonese have new opportunities for revitalization. The objective is to understand how new technologies and the Internet positively impact on its visibility and contribute to prevent their decline. To this end, the research, which is eminently qualitative, is conducted from two different approaches. From a communicative perspective, the aim is to discover the presence, use and spread of Aragonese in virtual platforms. Secondly, from an educational perspective, this research analyzes the possibilities of learning this language in these digital spaces. Our results show that the
\end{abstract}


estos entornos digitales. Nuestros resultados ponen de manifiesto que la lengua aragonesa está cada vez más presente en la Red y que en los últimos años ha visto incrementada su presencia en plataformas y herramientas virtuales, que pueden facilitar su visibilidad y dignificación. Por contra, su presencia en medios de comunicación online es muy escasa. En la misma línea, se observan reducidas oportunidades para aprender la lengua a través de la Red, tarea manifiestamente laboriosa y complicada por el reducido número de webs donde hallar recursos educativos digitales.

Palabras clave: NTIC, medios sociales, entornos virtuales de aprendizaje, lengua aragonesa, revitalización lingüística.
Aragonese language is increasingly present on the Internet and that in recent years its presence in virtual platforms has increased, which can dignify it and facilitate its visibility. In contrast, its use in digital media is very low. In the same vein, this study observes that there are few opportunities to learn this language online. This task is certainly laborious and complicated due to the limited number of websites where digital educational resources can be found.

Keywords: ICT, social media, virtual learning environments, minority languages, language revitalization.

\section{INTRODUCCIÓN}

Actualmente se hablan alrededor de 6.000 lenguas en el mundo (UNESCO, 2010). Esta diversidad cultural requiere ser valorada y protegida, más aún cuando existe una situación crítica para un conjunto de lenguas minoritarias en peligro de desaparición. Precisamente, la lengua aragonesa es una de las aproximadamente 2.500 lenguas amenazadas que la UNESCO recoge en la última edición de su Atlas de las Lenguas del Mundo en Peligro (2010). La situación de la lengua aragonesa resulta especialmente preocupante y merece ser objeto de investigación desde diferentes áreas con el fin de describir con la mayor exactitud posible su situación actual y plantear medios para revitalizarla.

Sin embargo, gracias a las nuevas herramientas tecnológicas de la información y la comunicación (NTIC, en adelante), el nacimiento de Internet, la rápida expansión de la Web 2.0 y el profundo cambio del ecosistema mediático y comunicacional derivado de estos procesos (Gil-Ramírez y Gómez de Travesedo-Rojas, 2018), las lenguas minoritarias como el aragonés pueden contar con nuevas oportunidades para su conservación y refuerzo. Los hablantes de cualquier idioma pueden ahora convertirse en sujetos activos y creadores de contenido en la Red. En concreto, los hablantes de la lengua aragonesa disponen de un conjunto modesto, pero cada vez más amplio, de entornos y herramientas virtuales para la difusión y el aprendizaje de esta lengua. En este contexto, resulta esencial conocer en qué medida las nuevas tecnologías y la expansión de la Web 2.0 pueden influir en su revitalización y cómo se está avanzando para conseguirla.

Diversas instituciones internacionales como la UNESCO (2010) y el Consejo de Europa (1992), además de autores reconocidos como Crystal (2000) o Moseley (2010), han alertado durante las últimas décadas sobre la situación de precariedad en la que se encuentra una buena parte de los idiomas del mundo; estimando que, a lo largo de este siglo, podrían desaparecer aproximadamente la mitad de estas lenguas. Este hecho resultaría de una extremada relevancia, no solo en el plano lingüístico, sino también en el cultural, e impactaría en aquellas sociedades y culturas minoritarias que podrían perder una parte fundamental de su identidad. En este sentido, Ovide (2008, p. 99) señala que "las lenguas son probablemente la seña de identidad más importante de una cultura, ya que codifican la realidad y la percepción del 
mundo de sus hablantes". En la misma línea, Crystal (2000, p. 98-99) manifiesta la necesidad de promover una mayor concienciación hacia esta problemática, ya que nos encontramos ante un desafío global que conviene afrontar, de modo que "todas las lenguas minoritarias y en peligro se beneficiarán de un aumento de la consciencia universal sobre diversidad lingüística". Este mismo autor, partiendo de un cuerpo de datos obtenidos en diferentes proyectos sobre mantenimiento de lenguas, propone seis factores que podrían reconocerse para una teoría sobre revitalización lingüística basada en las ideas de: (1) prestigio, (2) bienestar, (3) poder legítimo, (4) representación educativa, (5) capacidad de expresión escrita y (6) uso de tecnología electrónica por parte de la comunidad de hablantes.

Tabla 1

Factores-postulados para la revitalización de las lenguas minoritarias

\begin{tabular}{|c|l|}
\hline $\mathbf{1}$ & $\begin{array}{l}\text { Factores-postulados de Crystal (2000) } \\
\text { Una lengua en peligro progresará si sus hablantes aumentan su prestigio dentro de la } \\
\text { comunidad dominante. }\end{array}$ \\
\hline $\mathbf{2}$ & $\begin{array}{l}\text { Una lengua en peligro progresará si sus hablantes aumentan su bienestar en relación con } \\
\text { la comunidad lingüística dominante. }\end{array}$ \\
\hline $\mathbf{3}$ & $\begin{array}{l}\text { Una lengua en peligro progresará si sus hablantes aumentan su poder legítimo a los ojos } \\
\text { de la comunidad dominante. }\end{array}$ \\
\hline $\mathbf{5}$ & $\begin{array}{l}\text { Una lengua en peligro progresará si sus hablantes tienen una representación fuerte en el } \\
\text { sistema educativo. }\end{array}$ \\
\hline $\mathbf{6}$ & $\begin{array}{l}\text { Una lengua en peligro progresará si sus hablantes pueden escribirla. } \\
\text { en dicha lengua. }\end{array}$ \\
\hline
\end{tabular}

Fuente: Crystal (2000).

Por su parte, Ovide (2008) propone cinco grandes tareas para la recuperación de lenguas y culturas amenazadas, y cómo las tecnologías de la información y la comunicación pueden contribuir en cada una de ellas: (1) documentación, (2) difusión, (3) comunicación, (4) formación y (5) comercialización.

Tabla 2

Tareas para la recuperación de las lenguas minoritarias

\begin{tabular}{|c|l|l|}
\hline & Tarea & Contribución de las NTIC \\
\hline $\mathbf{1}$ & Documentación & $\begin{array}{l}\text { Obtención de los datos y su grabación en diferentes formatos y } \\
\text { soportes a fin de contar con evidencias reales de dicha lengua. }\end{array}$ \\
\hline $\mathbf{2}$ & Difusión & $\begin{array}{l}\text { Publicación de los contenidos en la Red a través de plataformas } \\
\text { digitales y de forma fácilmente accesible. "Si no estamos en } \\
\text { Internet, sencillamente no existiremos para la inmensa mayoría del } \\
\text { mundo" (Ovide, 2008, p. 104). }\end{array}$ \\
\hline $\mathbf{3}$ & Comunicación & Creación de una comunidad virtual en la que los hablantes de una \\
\hline
\end{tabular}




\begin{tabular}{|c|l|l|}
\hline $\mathbf{4}$ & Formación & $\begin{array}{l}\text { lengua amenazada puedan comunicarse e interactuar. } \\
\text { Expansión del conocimiento sobre la cultura y la lengua amenazada } \\
\text { a través de la Red de forma accesible para toda la población. }\end{array}$ \\
\hline $\mathbf{5}$ & Comercialización & $\begin{array}{l}\text { Concienciación de los hablantes sobre la defensa de su lengua, con } \\
\text { el fin también de atraer a nuevos hablantes potenciales. }\end{array}$ \\
\hline
\end{tabular}

Fuente: Ovide (2008).

Sin duda, todas estas acciones de revitalización se han visto favorecidas desde la llegada de la Web 2.0. Los hablantes de cualquier lengua pueden tener acceso, siempre que dispongan de los medios necesarios, a una gigantesca red donde pueden crear su propio espacio de convivencia y participación digital en su idioma. En esta línea, Belmar y Glass (2019) profundizan en el concepto de comunidad virtual como refugio para las diferentes especies lingüísticas del mundo y señalan que estas comunidades digitales pueden ser "la herramienta perfecta para que las lenguas minoritarias reclamen su propio espacio, dentro del sálvese quien pueda que es Internet" (Belmar y Glass, 2019, p. 15). La presencia digital "es un componente clave para el empoderamiento de comunidades lingüísticas minorizadas alrededor del mundo" (Belmar, 2020, p. 117), por lo que Internet y las redes sociales deben ser reconocidos como herramientas para programas de revitalización lingüística (Ovide, 2008; Crystal, 2000; Paricio-Martín y Martínez-Cortés, 2010; Campos-Bandrés, 2015; Belmar y Glass, 2019).

Como señalamos anteriormente, el aragonés es uno de estos idiomas minoritarios. Se trata de una lengua romance que todavía se habla en diferentes zonas, especialmente en el norte de Aragón. Está reconocida como lengua propia de esta comunidad autónoma, tal como se recoge en su Estatuto de Autonomía (2007) y en la vigente Ley de Lenguas de Aragón (2013). En la actualidad, se mantiene viva, aunque debilitada y amenazada. Según datos obtenidos del Censo de Población y Viviendas de 2011 del INE (Instituto Nacional de Estadística), el número de hablantes de aragonés sería de aproximadamente 25.000 personas. Si incluimos también a quienes lo pueden entender, esta cifra podría elevarse hasta las 45.000. Se trata de una de las lenguas amenazadas que la UNESCO recoge en la última edición de su Atlas de las Lenguas del Mundo en Peligro (2010). El Consejo de Europa (1992) la considera como una de las unique minority languages, ya que se utiliza exclusivamente en un territorio muy reducido.

\section{OBJETIVOS Y MÉTODO}

El objetivo principal de esta investigación será comprender cómo influyen las nuevas plataformas y herramientas virtuales en la difusión, el uso y el aprendizaje de la lengua aragonesa. De este primer objetivo derivan los siguientes, más específicos: (1) analizar su presencia y uso en diferentes plataformas digitales e interpretar la influencia de estos recursos en la revitalización de esta lengua minoritaria, (2) localizar plataformas digitales relacionadas con la lengua aragonesa y describir su contribución a la revitalización de este idioma, (3) producir una lista de herramientas educativas virtuales con recursos disponibles para su aprendizaje, (4) conocer el nivel de integración de las tecnologías de la información y la comunicación en la enseñanza del aragonés y (5) exponer las opciones formativas en modalidad online que existen en la enseñanza de la lengua aragonesa. 
Para la ejecución de esta investigación, se han combinado dos técnicas de tipo cualitativo: la entrevista semiestructurada y la observación no participante. En primer lugar, se realizaron entrevistas a un total de 14 informantes clave, expertos en lengua aragonesa con diferentes perfiles en el ámbito de la gestión, la formación (en todos los niveles), la comunicación en entornos virtuales y el tejido asociativo vinculado con nuestro objeto de estudio. Los perfiles de los sujetos entrevistados fueron los siguientes:

-Director del Centro Aragonés de Tecnologías para la Educación (CATEDU).

-Director General de Política Lingüística del Gobierno de Aragón.

-Jefe de Servicio de la Dirección General de Política Lingüística (DGPL, en adelante) del Gobierno de Aragón.

-Doctora en Filología Hispánica, asesora docente de la DGPL del Gobierno de Aragón y profesora de la Universidad de Zaragoza (Diploma de Filología Aragonesa y mención de Lengua Aragonesa en los grados de Magisterio).

-Doctora en Educación, directora del Diploma de Especialización en Filología Aragonesa de la Universidad de Zaragoza, investigadora y profesora de la Universidad de Zaragoza.

-Experto en radio y comunicación digital.

-Maestra perfil 1. Especialista en lenguas: inglés, francés y aragonés.

-Maestro perfil 2. Maestro de aragonés en Infantil y Primaria.

-Profesor perfil 3. Profesor de aragonés en Secundaria.

-Portavoz de la Asociación Cultural Nogará-Religada.

-Portavoz Asociación Cultural Consello d'a Fabla Aragonesa.

-Youtuber e instagramer de referencia para el aragonés.

-Responsable de la web de las Lenguas de Aragón.

-Miembro de la organización Softaragonés e investigador sobre el aragonés.

Asimismo, se ha hecho uso de métodos observacionales (observación sistematizada no participante) sobre plataformas virtuales generales y específicas relacionadas con la lengua aragonesa, así como portales y herramientas educativas virtuales dedicados al aragonés. En concreto, los ámbitos de observación analizados fueron: (1) lenguas minoritarias en la Red, (2) webs y blogs en aragonés, (3) asociaciones por el aragonés en la Red, (4) instituciones aragonesas en la Web, (5) medios digitales, (6) herramientas lingüísticas y educativas, (7) redes sociales, (8) aplicaciones para dispositivos móviles. Se utilizó un diario de campo para recoger la información necesaria a fin de alcanzar los fines propuestos.

Los resultados producidos con ambas técnicas de investigación (entrevistas y observación no participante) fueron articulados mediante complementación, una estrategia que combina los resultados procedentes de diferentes instrumentos de investigación cuando éstos no guardan entre sí relaciones jerárquicas ni de dependencia, sino que tienen una relevancia similar para la consecución de los objetivos del estudio (Callejo y Viedma, 2005).

El trabajo de campo no ha estado exento de dificultades, que derivan en una serie de limitaciones que esta investigación presenta. En primer lugar, solo ha sido posible el análisis de las herramientas educativas más populares entre los docentes y aquellas cuyos contenidos se presentan accesibles en abierto. Por otro lado, cabe señalar que las plataformas y herramientas virtuales observadas se encuentran en continua actualización de contenido; por 
lo que es posible que se hayan producido algunos cambios desde el periodo de análisis de este trabajo, que se prolongó durante seis meses, desde enero hasta junio de 2021.

\section{RESULTADOS}

\subsection{Presencia y uso del aragonés en la Red}

La presencia del aragonés en la Red data de 1994, gracias a la creación de la página web de la Asociación Cultural Nogará-Religada. Si aplicamos los postulados de Crystal (2000), observamos que uno de los puntos fuertes para su revitalización está relacionado con el avance en materia de herramientas digitales para esta lengua, gracias al esfuerzo de iniciativas particulares y públicas. La progresiva incorporación del aragonés al sistema educativo y las nuevas modalidades de formación online también suponen un gran aliento para su conservación, junto con la importante contribución para su defensa y visibilización por parte de asociaciones, colectivos y del activismo por la lengua aragonesa, que actúan tanto en entornos presenciales como en la Red. Sin embargo, la carencia de una grafía y gramática común pone en riesgo su potencial expansión. Otros obstáculos son la escasa financiación dedicada, así como su limitada presencia en medios de comunicación digitales, como se expondrá más adelante.

En cuanto a las tareas que propone Ovide (2008) para la recuperación de lenguas y culturas amenazadas (en relación con las NTIC), podríamos considerar que algunas de estas tareas han sido realizadas en el caso del aragonés; por ejemplo, el proceso de documentación y de difusión. En cambio, a pesar de los avances que se han materializado en los últimos años, existen otras tareas que todavía pueden ser mejoradas, tales como la creación de una comunidad robusta en la Red, el diseño de planes de formación online y la colaboración para la difusión de campañas de concienciación.

\subsubsection{El aragonés en webs y medios digitales}

En este apartado se analizarán en detalle los espacios virtuales donde existe presencia y uso del aragonés, tanto en webs de instituciones y asociaciones como en medios de comunicación. En concreto, se expondrán los (1) espacios online de proyectos internacionales, (2) webs de instituciones y asociaciones y (3) medios de comunicación digitales.

\subsubsection{Proyectos internacionales}

En este ámbito, destacan el Atlas de las Lenguas del Mundo en Peligro dentro de la web de la UNESCO, donde la lengua aragonesa es catalogada como "definitely endangered" ("claramente en peligro"); el espacio web de la campaña Diversidad Lingüística de la Comisión Europea (2012-2013), donde también se hace referencia al aragonés; y otros proyectos europeos sobre educación multilingüe y aprendizaje de lenguas, como el que desarrolla Mercator (alojado en la Fryske Akademy). Desde la web de este último proyecto, se accede a una wiki sobre lenguas minoritarias europeas, un mapa, así como dosieres sobre la situación educativa de algunas de estas lenguas (donde la aragonesa está incluida).

Por otra parte, el Proyecto Idiomas en Peligro de Extinción (ELP-Endangered Languages Project) consiste en una iniciativa global cuyo espacio online presenta información sobre las lenguas en vías de desaparición a nivel internacional. Destaca su mapa interactivo y la elevada cantidad de recursos audiovisuales que ofrece sobre estas lenguas. También resulta relevante el proyecto 
POCTEFA-LINGUATEC, financiado por la Unión Europea con la participación del Gobierno de Aragón, cuyo objetivo es desarrollar y difundir nuevos recursos, herramientas y aplicaciones lingüísticas para la digitalización del aragonés y otras lenguas como el euskera y el occitano.

\subsubsection{Webs de instituciones y asociaciones}

Uno de los espacios virtuales más destacados en torno al aragonés es la Web de las Lenguas de Aragón. Se trata de una iniciativa institucional, gestionada por la DGPL del Gobierno de Aragón con el objetivo de proteger, promocionar y difundir las lenguas propias de Aragón (aragonés y catalán de Aragón). Esta web contiene un elevado volumen de información, recursos y enlaces de interés. Entre otras opciones, es posible consultar el Archivo Audiovisual del Aragonés y acceder a reportajes sobre esta lengua. Asimismo, concentra y organiza diferentes proyectos e iniciativas (por ejemplo, el proyecto Aragón $3 \mathrm{~L}$ o la iniciativa Agora x I'aragonés, que cuenta con un blog vinculado), además de alojar el portal de la radio en aragonés Chisla Radio y enlazar diversas herramientas lingüísticas en aragonés (Aragonario y Traduze) y su canal de YouTube. Según fuentes de la DGPL del Gobierno de Aragón, durante el año 2020 el número de visitas que recibió esta web fue de 641.548, con un total de 117.778 visitantes. Esta iniciativa, que nació en 2017, lleva camino de alcanzar en 2021 los 2 millones de consultas. También resulta interesante la actividad del portal Charrando, un espacio que actualmente se encuentra desactualizado, pero que tuvo gran relevancia en la década de los 2000.

El uso del aragonés en el conjunto de las webs institucionales de la región podría describirse como esporádico y anecdótico. Existe algún ejemplo destacable, como el portal de la Comarca Alto Gállego, que ofrece un repositorio con información sobre el aragonés del Alto Gállego o algunos apartados de la web de la Comarca Hoya de Huesca. Un panorama muy similar se da en los espacios web de las organizaciones políticas de Aragón. Solamente se ha encontrado presencia de esta lengua en cinco espacios: Chunta Aragonesista, Puyalón, Purna, Estado Aragonés/Estau Aragonés y el Sindicato Obrero Aragonés, aunque el uso de la lengua en estas sites es ciertamente limitado.

Sin embargo, existen diferentes espacios virtuales de asociaciones relacionadas con el aragonés donde esta lengua obtiene un mayor protagonismo y cuenta con más recursos y materiales accesibles por los usuarios. Por ejemplo, la web del Rolde de Estudios Aragoneses (REA) ofrece una perspectiva amplia de la cultura e identidad aragonesa. Destaca un mapa lingüístico de las lenguas minoritarias en Aragón, una unidad didáctica de acercamiento a la lengua aragonesa, un informe sobre el aragonés en el siglo XXI o la versión digital de la revista infantil Papirroi, escrita en aragonés.

La Academia de l'Aragonés, constituida por la asociación Estudio de Filología Aragonesa (EFA), ofrece prácticamente todos los textos de su web en esta lengua. Es reseñable la colección EDACAR (Ediciones Digitales de la Academia del Aragonés), así como las publicaciones digitalizadas de todas las colaboraciones en Heraldo Escolar (recursos escolares en aragonés). Por su parte, la Sociedat de Lingüistica Aragonesa (SLA) muestra, sobre todo, información sobre la propia asociación y sus estudios.

\subsubsection{Medios de comunicación digitales}

El número de medios de comunicación digitales (diarios digitales, radios online, programas radiofónicos y programas televisión) con presencia del aragonés es muy reducido. El diario 
Arredol fue el primer diario digital en aragonés, creado en 2011 y cerrado en 2016. La primera página de información exclusivamente en aragonés con presencia en Internet fue ARebista.net, una publicación digital quincenal lanzada en el año 2000. Actualmente, no existe ningún diario digital específicamente en este idioma. El diario Aralnfo dedica algún espacio en su web a esta lengua, sobre todo en las entradas que tratan sobre las lenguas de Aragón. Asimismo, la sección Heraldo Escolar del Heraldo de Aragón incluye desde 2013 (en su versión física y digital) una columna en esta lengua dedicada a recursos escolares.

La única emisora de radio online totalmente en aragonés es Chisla Radio. Se trata de un proyecto impulsado por la DGPL del Gobierno de Aragón desde 2017, que ofrece sus programas en formato podcast a través de la plataforma iVoox. Apenas existe variedad en su programación: solo cinco espacios con un número muy limitado de episodios. Otras radios independientes han emitido (o emiten) programas en esta lengua, como es el caso de Radio Topo, que contaba en su parrilla con los espacios Fendo Orella y A Hora Charrante, todavía disponibles en su web. El programa Tierra de Barrenaus (aún en emisión) es un espacio de radio online con una larga trayectoria, que cuenta con numerosos episodios y un blog vinculado. Radio La Granja también cuenta en su parrilla con el programa O Suenio d'o Dragón, disponible en streaming y en formato podcast.

En cuanto a la televisión, destaca Charrín Charrán, el primer programa en aragonés de Aragón $T V$. Sus capítulos están disponibles a la carta desde la web de este medio.

\subsubsection{Herramientas lingüísticas digitales y aplicaciones móviles}

La gran mayoría de instrumentos de este tipo se ha desarrollado en los últimos años gracias a la actividad de la organización Softaragonés y a la DGPL del Gobierno de Aragón. Anteriormente habían existido iniciativas como FablaGNU o laragonesenorete que consiguieron, entre otros logros, que el aragonés figurara en los listados de códigos ISO, aspecto que ha facilitado los trabajos posteriores en este ámbito.

El Aragonario es un diccionario bilingüe aragonés-castellano online con doble sentido de búsqueda. Según sus propios gestores, esta herramienta recibió en el año 2020 alrededor de 83.000 visitas y 38.000 visitantes. Se trata de un proyecto en continua actualización y mejora que comenzó con unas 20.000 entradas en castellano-aragonés y que, ahora, ha superado la cifra de 50.000. En la nueva versión del Aragonario, se ha incluido el Conchugador, una herramienta que ofrece modelos de conjugación de verbos siguiendo los patrones del aragonés. Estrechamente ligado al Aragonario se encuentra el traductor bilingüe castellanoaragonés denominado Traduze, que permite traducir textos, documentos completos y páginas web. Esta herramienta persigue un objetivo similar a un proyecto anterior desarrollado por Juan Pablo Martínez (con el apoyo de Softaragonés) dentro de la comunidad de Apertium. Se trata de un traductor trilingüe que trabaja con textos, documentos y páginas web en las tres lenguas de Aragón (castellano, aragonés y catalán).

En otro orden, el Tresoro d'a Luenga Aragonesa es una recopilación de términos aragoneses incluidos en diferentes repertorios léxicos. En la Red podemos encontrar la edición digital de este diccionario, alojado dentro de la sección de patrimonio lingüístico de la web del SIPCA (Sistema de Información del Patrimonio Cultural Aragonés).

La Biquipedia (Wikipedia en aragonés) es una enciclopedia libre impulsada desde la 
organización Softaragonés. Se nutre de contenido propio, creado en aragonés de forma colaborativa entre los usuarios que participan en esta wiki. Existen casi 40.000 artículos disponibles en aragonés, que sitúan a este recurso en la posición 103 del ranking global de wikipedias, justo por delante de la versión en kurdo y 10 puestos por debajo de la propia del gaélico/irlandés.

Por otro lado, la organización Softaragonés es la responsable de la adaptación de una amplia variedad de software, que puede descargarse a través de su propia web. Algunos ejemplos de recursos informáticos actualizados disponibles actualmente en aragonés son Firefox y LibreOffice, el corrector ortográfico de Hunspell (empleado en LibreOffice, Thunderbird, Firefox o Linux) y eSpeak (sintetizador de voz), además de poder emplear el aragonés como lengua vehicular en Moodle, MediaWiki o Scratch, entre otras plataformas y programas. En cuanto a las aplicaciones para dispositivos móviles, la mayor parte están enfocadas a la comunicación más que al aprendizaje de la lengua y han sido desarrolladas e impulsadas, principalmente, por la organización Softaragonés o por la DGPL de Aragón. En la tabla 3 se recogen las apps más relevantes, indicando sus creadores y los sistemas operativos donde están disponibles.

Tabla 3

Aplicaciones para dispositivos móviles en aragonés

\begin{tabular}{|c|c|c|c|}
\hline & Aplicación & Android & iOS \\
\hline \multirow{5}{*}{ 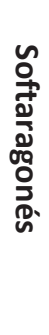 } & Opción de usar el navegador Firefox en aragonés. & $\mathrm{X}$ & $\mathrm{X}$ \\
\hline & Opción de usar la red social Telegram en aragonés. & $\mathrm{x}$ & $\mathrm{x}$ \\
\hline & Swiftkey: teclado predictivo en aragonés. & $\mathrm{x}$ & $\mathrm{x}$ \\
\hline & Lichess: para jugar al ajedrez en aragonés. & $\mathrm{x}$ & $\mathrm{x}$ \\
\hline & Birthdays y Birthday Calendar: recordatorios de cumpleaños & $x$ & \\
\hline \multirow{4}{*}{ 뭄 } & $\begin{array}{l}\text { Ilesias del Sarrablo: ofrece información sobre las famosas iglesias de la } \\
\text { zona del Serrablo (Huesca). }\end{array}$ & $x$ & $\mathrm{x}$ \\
\hline & $\begin{array}{l}\text { Camino de Santiago en Aragón: información sobre la ruta del Camino de } \\
\text { Santiago a su paso por Aragón. }\end{array}$ & $x$ & $\mathrm{x}$ \\
\hline & $\begin{array}{l}\text { LiteARAtura: información acerca de la vida y obra de autores de la } \\
\text { literatura en aragonés. Existen varias obras disponibles online. }\end{array}$ & $\mathrm{x}$ & \\
\hline & $\begin{array}{l}\text { Agora por l'aragonés: enlaces a herramientas y recursos online, así } \\
\text { como información sobre los colaboradores de esta iniciativa. }\end{array}$ & $\mathrm{x}$ & \\
\hline \multirow{3}{*}{ 온 } & Teclado predictivo de Google (Gboard). & $X$ & \\
\hline & Softcatalà: traductor aragonés-catalán (y catalán-aragonés). & $x$ & $\mathrm{x}$ \\
\hline & $\begin{array}{l}\text { Aragonese-Spanish Translator: traductor castellano-aragonés (en doble } \\
\text { dirección). }\end{array}$ & $x$ & \\
\hline
\end{tabular}

Fuente: elaboración propia.

\subsubsection{El aragonés en las redes sociales}

En este apartado se analizará la presencia y uso del aragonés en las principales redes sociales: 
Facebook (grupos, páginas y perfiles), Instagram (hashtags y perfiles), Twitter (hashtags y perfiles), además de YouTube y Vimeo.

En Facebook existen diferentes grupos (públicos y privados), donde los usuarios emplean con frecuencia esta lengua. En general, sus miembros suelen compartir enlaces de interés sobre el aragonés (información, noticias, vídeos, imágenes...) y comentan noticias de carácter político, social o académico sobre su situación. Tres ejemplos de grupos activos son: Tradizions y paroletas d'o nuestro Aragón; Aragonés: actividatz y recursos; y Aragonés: charrar, ragonar, parlar, fablar... Este último grupo ha sido analizado por Belmar (2020), quien lo considera un ejemplo de comunidad virtual que sirve de refugio para una lengua minoritaria, en este caso, la aragonesa.

En Facebook también se puede hallar una presencia considerable a través de páginas y, sobre todo, perfiles. En la mayoría de los casos observados, la lengua se emplea con tres intenciones: en primer lugar, para la difusión de información de carácter lingüístico, cultural e histórico; por otra parte, para reflexionar sobre la propia lengua; $y$, en menor grado, los usuarios también la usan para hablar de temas cotidianos y de experiencias personales.

En la red social Instagram, el hashtag con más actividad es \#aragones (más de 8.300 publicaciones). Junto a su variante con tilde (\#aragonés), suman más de 11.000 publicaciones en total (en junio de 2021). Otro hashtag muy recurrido es \#lenguaaragonesa (con más de 1.000 publicaciones), mientras que su variante en aragonés (\#luengaaragonesa) tiene unas 230. Existen algunos hashtags específicos para las variedades dialectales del aragonés, por ejemplo: \#cheso (1.949 publicaciones), \#patués (121), \#chistabin (59) o \#ansotano (50).

Entre los perfiles, encontramos la presencia de varios influencers que utilizan el aragonés y que realizan una labor muy relevante en su visibilización debido a la calidad de su contenido y repercusión. La mayoría de ellos utiliza esta lengua con el fin de visibilizarla y difundirla, tratando sobre distintas temáticas: experiencias personales, humor, creación literaria, recursos didácticos, eventos y noticias. Predominan los contenidos sobre asuntos cotidianos, a fin de atraer al público joven y motivarles a aprender y defender la lengua. Destacan, por ejemplo, los contenidos que difunde en sus posts y stories la influencer Silvia Cebolla (@silvia_cebolla), los posts en clave de humor de Memes en aragonés (@memes.en.aragones) y Ribagorzan memes (@ribagorzan_memes), el contenido didáctico de En patués (@en.patues), además del perfil del programa Charrín Charrán o los de asociaciones culturales (como el Consello d'a Fabla Aragonesa, Nogará-Religada o el Ligallo de Fabláns) e instituciones formativas (Diploma Filología Aragonesa).

En Twitter, el hashtag con más actividad es \#aragones. Cuenta con una gran variedad temática: noticias y artículos sobre el aragonés y la identidad aragonesa, creación literaria y vídeos de programas de televisión. Otros hashtags relevantes son: \#aragonesoficial, \#lenguaaragonesa, \#luengaaragonesa, \#vivirenaragones o \#aragonesofizial, así como aquellos que hacen referencia a sus variedades dialectales. En esta red social, es saliente el perfil de Jorge Pueyo (@jorge_pueyo95), que ofrece un diario matinal en aragonés, además de otros perfiles activos como el del programa Charrín Charrán.

Por otra parte, las plataformas digitales de contenidos audiovisuales como YouTube y Vimeo representan una oportunidad para difundir esta lengua a través de un contenido cada vez más demandado. YouTube ofrece una gran variedad de recursos, algunos de gran valor e interés 
educativo. Cuenta con un amplio repositorio de vídeos de diversas temáticas y con importantes posibilidades educativas. En muchas ocasiones, este material audiovisual es compartido a través de las redes sociales anteriormente referidas. Sin embargo, en YouTube encontramos un escaso número de canales dedicados expresamente a la enseñanza del aragonés (Parola-Paroleta, el canal de Javier Vispe y Millor en Aragonés, básicamente); aunque existen espacios de carácter más general que ofrecen recursos muy interesantes, por ejemplo, el canal de las Lenguas de Aragón o el del programa Charrín Charrán (la sección Pizarra y clarión). En la tabla 4 se exponen algunos ejemplos de canales de YouTube, organizados por las temáticas más frecuentes.

Tabla 4

Ejemplos de canales en aragonés en YouTube

\begin{tabular}{|l|l|l|}
\hline \multicolumn{1}{|c|}{ Comunicación y difusión } & \multicolumn{1}{|c|}{ Entretenimiento } & \multicolumn{1}{c|}{ Educación } \\
\hline Lenguas de Aragón & Silvia Cebolla & Parola-Paroleta \\
Charrín Charrán & Cintas d'Enguero & Javier Vispe \\
Iris O. Campos Bandrés & Aguarash Entertainment & Millor en Aragonés \\
Nogará & Jorge Pueyo & \\
Barrenau & Roberto Rodes & \\
Marco A. Joven Romero & & \\
Charrando TB & & \\
\hline
\end{tabular}

Fuente: elaboración propia.

\subsection{Aprendizaje del aragonés a través de entornos y herramientas digitales}

\subsubsection{Entornos virtuales de aprendizaje}

Recursos en línea para el aragonés es la principal base de herramientas y recursos didácticos online para el aprendizaje de esta lengua. Gestionada por la DGPL, ofrece una gran variedad de materiales: enlaces a herramientas lingüísticas (Aragonario y Traduze), documentos para descargar (criterios lingüísticos, impresos en aragonés o exámenes de nivel) y una serie de recursos didácticos que pueden servir como material educativo para docentes o para el aprendizaje de la lengua de forma autodidacta a través de la Red. Dentro de este conjunto de recursos, es prominente el amplio abanico de materiales digitalizados (manuales, propuestas didácticas o cuentos) disponibles para descargar. Sin embargo, apenas existe posibilidad de interacción entre usuarios a través de estos recursos (solo el programa Viene-ven y algunas actividades muy concretas del proyecto Aragón $3 L$ ofrecen esta opción, en un nivel muy básico).

A esta plataforma podemos añadir los contenidos y recursos educativos ofrecidos en las webs de las principales asociaciones del aragonés. Asimismo, los blogs pueden resultar espacios útiles para el aprendizaje; sobre todo, aquellos dedicados expresamente a la enseñanza de la lengua. Merecen especial atención dos ejemplos. Por un lado, el blog L'aragonés en a escuela, especializado en cuentos y relatos; así como Parolero que, además de ofrecer numerosos recursos y propuestas educativas, dispone de un enlace a un Padlet (un muro digital) con abundante material. Otro blog que ofrece un contenido reseñable es Millor en Aragonés, un 
espacio sin actualizar desde 2014, pero donde todavía se encuentran accesibles materiales para aprender aragonés; destacando los vídeos de la serie Aprende aragonés con Totón, disponibles también en YouTube.

\subsubsection{Uso de plataformas y herramientas digitales en la enseñanza formal y no formal}

Una vez expuestos los entornos de aprendizaje, se abordará el uso de estas plataformas y herramientas en el aprendizaje del aragonés en (1) los espacios de enseñanza formal (los colegios e institutos de Educación Primaria y Secundaria de la comunidad autónoma y la Universidad de Zaragoza) y (2) en los contextos de educación no formal.

\subsubsection{Colegios e institutos de Aragón}

Desde el curso 1997-1998 existe una enseñanza reglada del aragonés en la escuela pública de Aragón. Según datos de la DGPL, en el curso 2020-2021, fueron un total de 26 los centros educativos que impartieron la materia de lengua aragonesa, con un alumnado total de 1.097 estudiantes. Diferentes expertos en materia de educación y aragonés consultados señalan que la aplicación de las NTIC en el aula depende en gran medida del perfil docente y de diversos factores personales. Asimismo, en el caso de la enseñanza del aragonés puede influir también la visión que se tenga del ámbito de aplicación de la propia lengua.

Sobre el uso de las nuevas tecnologías para el aprendizaje del aragonés en las aulas, la maestra de perfil 1 participante en nuestras entrevistas (especialista en inglés, francés y aragonés) afirma que las NTIC pueden ser un buen instrumento para darle una perspectiva diferente a las materias lingüísticas, siempre con una finalidad comunicativa, además de una gran herramienta motivacional. Por otra parte, el docente de perfil 2 (maestro de aragonés en Primaria e Infantil), considera que las NTIC pueden facilitar el aprendizaje de aragonés, destacando su valor lúdico. El profesor de perfil 3 (profesor de aragonés en Secundaria) las utiliza con frecuencia porque, opina, ayudan a mejorar la competencia comunicativa y la integración del alumnado en comunidades virtuales de hablantes.

Son una herramienta maravillosa para poder darle un enfoque diferente a las asignaturas lingüísticas (Maestra perfil 1).

Yo utilizo las nuevas tecnologías como enganche, como motivación. Creo que son una gran herramienta motivacional (Maestra perfil 1).

El uso de las NTIC puede resultar muy lúdico y además sirve para repasar vocabulario y conceptos de sesiones anteriores (Maestro perfil 2).

Ayudan a mejorar la competencia comunicativa y a integrar al alumno en una comunidad hablante, aunque sea virtual (Profesor perfil 3).

En cuanto a los recursos utilizados, los expertos entrevistados señalan la relevancia de los libros o materiales impresos que han pasado por procesos de digitalización. Estos expertos defienden el aumento progresivo de recursos y materiales digitales que pueden utilizarse en las aulas.

Desde el punto de vista didáctico, o sea, pensados para el aula, creados como material didáctico... la mayor parte de lo que hay son libros o material impreso que se ha transformado en PDF (Experta en lengua aragonesa y profesora de la Universidad de Zaragoza). 
Existen muchísimos materiales digitales que se pueden utilizar. El repositorio ahora es amplísimo (Experta en lengua aragonesa y profesora de la Universidad de Zaragoza).

Ahora hay muchos más recursos. Pero es difícil competir con los materiales y recursos de lenguas mayoritarias como el castellano o el inglés (Asesora docente DGPL y profesora de la Universidad de Zaragoza).

En concreto, las herramientas y recursos digitales que los docentes de aragonés utilizan en sus clases - especialmente con el fin de aumentar la motivación del alumnado- son recursos con un alto componente lúdico. Habitualmente, los docentes tratan de compensar la carencia de materiales adaptados al nivel educativo creando los suyos propios con diferentes herramientas digitales. El profesor de perfil 3 indica que utiliza a menudo Internet, sobre todo algunas de las webs de referencia citadas en este trabajo. Además, emplea algunas herramientas virtuales y el blog. La maestra de perfil 1, por su parte, expone las ventajas del uso de redes sociales (como YouTube, Instagram, Twitter) para realizar proyectos en áreas de lengua (especialmente en niveles avanzados).

Por ejemplo, para crear un cuento con dibujos animados, lo que hago es descargarlo en español o inglés, quitarles el volumen a las voces humanas y redoblarlo en aragonés. Y lo mismo con canciones infantiles (Maestro perfil 2).

Utilizo a menudo Internet, Ahaslide y el blog (Profesor perfil 3).

Utilizamos Instagram como "contenedor" del trabajo que estábamos haciendo. Y lo que hacían ellos era leerlo y comentar en este perfil. El objetivo era enfocar la lengua como una manera de comunicar y que me comuniquen cosas que me interesan. (Maestra perfil 1).

\subsubsection{Universidad de Zaragoza}

En la Universidad de Zaragoza se imparte, desde el curso 2011-2012, el Diploma de Especialización en Filología Aragonesa en modalidad presencial. En el curso 2020-2021, se ofertó la modalidad online de este estudio. Según su directora, las herramientas virtuales van a tener un impacto muy significativo en el Diploma, con consecuencias positivas para la enseñanza, la investigación y la difusión del aragonés.

Las herramientas virtuales le van a dar al Diploma una nueva etapa y van a hacer que se abra a muchísima más gente (Directora del Diploma de Especialización en Filología Aragonesa).

Tambien en el curso 2020-2021, se ha puesto en marcha la mención en Lengua Aragonesa en los Grados de Magisterio de Primaria e Infantil. Este curso, debido a la situación sanitaria, se ha ofrecido la opción de seguir las sesiones teóricas en streaming, apostando así por un modelo mixto (blended learning). Dos de las profesoras entrevistadas que imparten docencia en estos estudios afirman que la expansión de la Web 2.0 ha servido para democratizar el acceso al aragonés y ha facilitado la autonomía de los estudiantes.

El mundo virtual en el caso de la enseñanza del aragonés juega un papel de democratización. Permite un acceso a muchas personas que, de otro modo, no podrían tener ningún recurso (Experta en lengua aragonesa y profesora de la Universidad de Zaragoza. 
Las nuevas tecnologías lo que permiten es una mayor autonomía del alumnado (Asesora docente DGPL y profesora de la Universidad de Zaragoza).

\subsubsection{Educación no formal}

La educación no formal ha sido durante mucho tiempo la única opción de aprender aragonés para las personas adultas. La asociación Nogará-Religada cuenta con una larga trayectoria en la enseñanza de esta lengua y, desde hace varios años, ofrece su formación también online. Otros colectivos, como el Consello d'a Fabla Aragonesa, a pesar de contar con una gran experiencia en la enseñanza de aragonés, no ofrecen formación a distancia. Por último, cabe citar una alternativa impulsada desde el ámbito institucional: los cursos de aragonés que se organizan periódicamente (actualmente en modalidad online) desde la Cátedra Johan Ferrández d'Heredia de la Universidad de Zaragoza y en colaboración con el Gobierno de Aragón.

\section{CONCLUSIONES}

El presente estudio observa que el aragonés está cada vez más presente en la Red. Esta lengua se encuentra en continua expansión a través del mundo digital. En los últimos años se ha visto incrementada su presencia en plataformas y herramientas virtuales y actualmente sigue ganando terreno, sobre todo, en las redes sociales. Se trata de una dinámica muy positiva, puesto que los expertos en la materia coinciden en señalar la importancia de la presencia de una lengua minoritaria en los espacios digitales para impulsar su revitalización. Sin embargo, el aumento del número de espacios de referencia online no significa que el aragonés haya alcanzado las metas deseables en este campo, pues los recursos son aún ciertamente limitados. Asimismo, este desarrollo digital no se ve tan acelerado en el ámbito de la educación virtual. Los espacios dedicados al aprendizaje online son mínimos y se concentran en unos pocos portales de referencia.

En todo caso, resulta más sencillo encontrar recursos en aragonés en el mundo digital que en los contextos analógicos. Gracias a los entornos virtuales, los hablantes de esta lengua cuentan ahora con más espacios donde utilizarla de forma diaria, integrándola en su vida, y donde poder socializar a través de una red de usuarios cada vez más amplia. Con este argumento, expertos en comunicación y lengua aragonesa afirman que es más fácil conectar con el aragonés en el mundo digital que en el espacio analógico.

En relación con la idea anterior, la expansión de la Web 2.0 ha contribuido a democratizar el acceso al aragonés. Internet ha facilitado la entrada a un espacio común de colaboración y participación social. En el caso de esta lengua, tanto sus hablantes como aquellas personas interesadas en conocerla disponen de una mayor variedad, número de recursos y espacios donde poder entrar en contacto con ella, siempre que cuenten con conexión a la Red.

Estas herramientas digitales pueden facilitar la dignificación de esta lengua. Durante siglos, el aragonés ha arrastrado una serie de estigmas y prejuicios. Al dotarla de una presencia observable (oral y escrita), las NTIC pueden ayudar a superar estos problemas y aumentar su prestigio, dado que el hecho de reflejar por escrito una lengua le confiere un cierto estatus. Este proceso de dignificación refuerza y motiva a los aprendices, además de mejorar la percepción que los hablantes experimentados tienen sobre ella.

Los grandes problemas para la difusión del aragonés en el mundo analógico se trasladan al 
mundo digital. Gran parte de los obstáculos detectados en el proceso de revitalización en la Red coinciden con aquellas problemáticas históricas que existen en el contexto analógico. Uno de los principales obstáculos es la falta de financiación y de apoyo institucional a su preservación y promoción. Otra de las grandes dificultades es la falta de consenso entre la comunidad de hablantes en torno a la grafía y la normalización de la lengua, lo que dificulta su enseñanza y difusión de forma coherente al no emplearse un modelo único. Este aspecto facilita la desmotivación de los nuevos usuarios potenciales. Esta falta de consenso provoca un desgaste a los hablantes y afecta negativamente a la imagen externa de la propia lengua. Por todo ello, desde diferentes ámbitos se apunta hacia el entendimiento y la confluencia como solución.

A pesar del terreno ganado en los espacios digitales en los últimos años, la presencia de esta lengua en los medios de comunicación digitales resulta muy escasa. No existe ningún diario digital íntegramente en aragonés, tan solo alguna sección y noticias puntuales en varios medios digitales. La única radio en aragonés, Chisla Radio, ofrece un contenido muy limitado. El número de programas de radio (accesibles en formato podcast o en streaming) es también muy bajo. En cuanto a la televisión, solo encontramos un programa accesible en la Red: Charrín Charrán. Expertos en comunicación expresan la necesidad de apostar por más programas en aragonés desde los medios públicos.

En la misma línea, hay pocas oportunidades para aprender la lengua a través de la Red. Es posible aprender aragonés en entornos virtuales, pero esta tarea puede ser manifiestamente laboriosa y complicada por el reducido número de webs donde hallar recursos educativos digitales. En este sentido, destaca la sección Recursos en línea de la web de las Lenguas de Aragón y algunos materiales ofrecidos en los portales de las principales asociaciones. Del mismo modo, apenas se observan blogs enfocados a la enseñanza. En cuanto a los recursos didácticos accesibles en las herramientas educativas virtuales observadas, las opciones también son muy escasas. Los docentes manifiestan que existe una falta de materiales digitales adaptados para la enseñanza de esta lengua. Por ello, uno de los principales retos sería cubrir esta necesidad de recursos.

Por el contrario, las redes sociales representan una oportunidad de encuentro y práctica entre hablantes y estudiantes. Destacan, en este ámbito, los grupos de Facebook donde se comparte información y recursos, así como algunos perfiles de Instagram y Twitter que difunden contenidos para la adquisición de competencias lingüísticas, derribando barreras temporales, culturales y espaciales (Iñesta-Fernández y Sixto-García, 2020).

Las NTIC están en proceso de integración en la enseñanza del aragonés, tanto en entornos formales como no formales. En la educación formal, el uso de las tecnologías digitales aplicadas a la educación depende, en gran medida, de cada docente y contexto. Sin embargo, los docentes que han participado en la investigación coinciden en señalar las ventajas que pueden aportar las plataformas y herramientas digitales en las clases de aragonés, entre ellas: (1) favorecer un ambiente positivo, (2) aumentar la motivación de los estudiantes, (3) ofrecer una variedad de posibilidades didácticas y (4) permitir el acceso a numerosas fuentes de información.

Finalmente, se considera que el futuro del aragonés en el contexto digital habría de construirse sobre los pilares de la concienciación, la colaboración y la educación. En primer lugar, es 
necesario motivar a los propios hablantes, involucrar a los nativos de la lengua, apostar por campañas de concienciación sobre el valor cultural del aragonés y fomentar su aprendizaje. El objetivo debería ser la creación de una comunidad de hablantes más amplia, robusta e interconectada. Para ello, la Web representa un espacio de oportunidades para desarrollar proyectos de revitalización, especialmente a través de las redes sociales y de forma conjunta entre instituciones y colectivos ciudadanos, sin olvidar que la educación constituye la mejor herramienta para que el aragonés sume hablantes. Resulta fundamental el fomento de su enseñanza de la forma más accesible, sencilla y motivadora posible. $Y$, de nuevo, la Red nos brinda un espacio idóneo para conseguirlo.

\section{Referencias}

Belmar, G. (2020). ¿Pueden las lenguas minorizadas respirar tranquilas en las redes sociales? El papel de las comunidades virtuales como refugios de uso a través de la observación de un grupo de Facebook para hablantes de aragonés. Études romanes de Brno, 1, 113-124. https://doi.org/10.5817/ERB2020-1-8

Belmar, G. \& Glass, M. (2019). Virtual communities as breathing spaces for minority languages: reframing language use in social media. Adeptus, 14, 1-24. https://doi.org/10.11649/a.1968

Callejo, J. y Viedma, A. (2005). Proyectos y estrategias de investigación social. Mc Graw Hill.

Campos-Bandrés, I.O. (2015). Las NTIC en la conservación de las lenguas minoritarias. Una investigación - acción en el ámbito educativo. UT. Revista de Ciències de l'Educació, 1, 64-82. https://cutt.ly/Smk5Mjz

Consejo De Europa (1992). Carta Europea de las Lenguas Minoritarias o Regionales. Recuperado de https://cutt.ly/PmkBFZZ

Crystal, D. (2000). Language Death. Cambridge University Press.

Gil-Ramírez, M. \& Gómez de Travesedo-Rojas, R. (2018). Uso del smartphone en el periodismo actual: ¿'hacia un nuevo modelo mediático? Estudio de caso del contexto malagueño. Ámbitos. Revista Internacional de Comunicación, 2(41), 5-21. https://cutt.ly/dR1IVIL

Iñesta-Fernández, N. \& Sixto-García, J. (2020). Redes sociales, convergencia y narrativas transmedia en la promoción de las Islas Canarias. Ámbitos. Revista Internacional de Comunicación, 48, 148170. https://doi.org/10.12795/Ambitos.2020.i48.08

Moseley, C. (ed.) (2010). Atlas de las Lenguas del Mundo en Peligro 2010. UNESCO.

Ovide, E. (2008). Desde la diversidad hacia la desigualdad: ¿destino inexorable de la globalización? Revista Electrónica Teoría de la Educación: Educación y Cultura en la Sociedad de la Información, 9(2), 5-18. https://cutt.ly/zmk52Mn

Paricio-Martín, S.J. \& Martínez-Cortés, J. P. (2010). Nuevas vías de revitalización para lenguas minorizadas: la repercusión de internet en el caso del aragonés. Digithum, 0(12), 1-11. https://cutt.ly/Wmk585c 